\title{
PLANIFICADOR ESTRATÉGICO PARA OPERACIONES DE RESCATE MEDIANTE VEHÍCULOS TERRESTRES NO TRIPULADOS
}

\author{
Manuel Toscano-Moreno, Anthony Mandow, María Alcázar Martínez y Alfonso García-Cerezo \\ Universidad de Málaga, Andalucía Tech, Departamento de Ingeniería de Sistemas y Automática \\ $\{$ m.toscano, amandow, mamartinezs, ajgarcia\}@uma.es
}

\section{Resumen}

El despliegue de plataformas robóticas como apoyo a la gestión de situaciones de emergencia constituye un reto que persigue mejorar la eficiencia en misiones de búsqueda y rescate. Este artículo propone un planificador estratégico para el rescate de victimas mediante un equipo de robots terrestres. Este sistema inteligente permitirá planificar las trayectorias para acceder a las victimas de acuerdo con su ubicación y prioridad de asistencia médica (triaje). Para la planificación se propone una nueva variante del algoritmo "fast marching method" (FMM). En este trabajo se ofrecen resultados preliminares mediante un ejemplo de aplicación en condiciones simuladas.

Palabras clave: Planificador estratégico, operaciones de rescate, algoritmo fast marching method, vehículos terrestres no tripulados, triaje.

\section{INTRODUCCION}

La respuesta en emergencias requiere una intervención rápida para la atención a las víctimas así como una reducción del riesgo para los miembros del equipo de rescate. En este sentido, el desarrollo de sistemas robóticos y mecatrónicos con ciertas capacidades inteligentes tiene un enorme potencial en escenarios de crisis [11]. Así, la integración de vehículos terrestres no tripulados (UGV, unmanned ground vehicle) en los equipos de búsqueda y rescate (SAR, search and rescue), puede ayudar al éxito de estas misiones [17].

Estos sistemas robóticos inteligentes se pueden beneficiar de recientes contribuciones en vehículos aéreos $[3,5]$ y terrestres rodados [10] o con patas [7], realidad aumentada [19], teleoperación [16], planificación de manipuladores móviles [22], control visual [18], sistemas hápticos [6], robots modulares [2], comunicaciones en equipos multi-robot [21], y redes de sensores [9].

Las misiones SAR se desarrollan básicamente en una secuencia de operaciones [14]: 1) Exploración del entorno, donde la información obtenida facilita la toma de decisión de las restantes operaciones;
2) búsqueda de posibles víctimas, donde se localizan y clasifican las víctimas atendiendo a criterios médicos; 3) rescate y atención médica a víctimas, donde se accede a las víctimas para estabilizarlas y extraerlas; y 4) evacuación de víctimas. En este trabajo, se aborda el tercero de estos puntos mediante UGV, con el objetivo de acceder a un conjunto de víctimas para prestar una primera atención, tal y como se ilustra en el ejemplo de la figura 1 .

El artículo introduce un nuevo planificador estratégico para operaciones de rescate y atención médica mediante un equipo de múltiples UGV. Se propone un sistema inteligente para planificar las trayectorias que deberán seguir los UGV con objeto de acceder a un conjunto de víctimas previamente localizadas y clasificadas mediante triaje médico. Este planificador multi-objetivo, además de la prioridad de asistencia médica asignada, considera el tiempo medio de espera por víctima mediante una variante anisotrópica del algoritmo "fast marching method" (FMM).

El resto del artículo se organiza de la siguiente manera. En la sección 2 se revisan trabajos previos. En la sección 3 se introduce el algoritmo estratégico propuesto. A continuación, en la sección 4 se

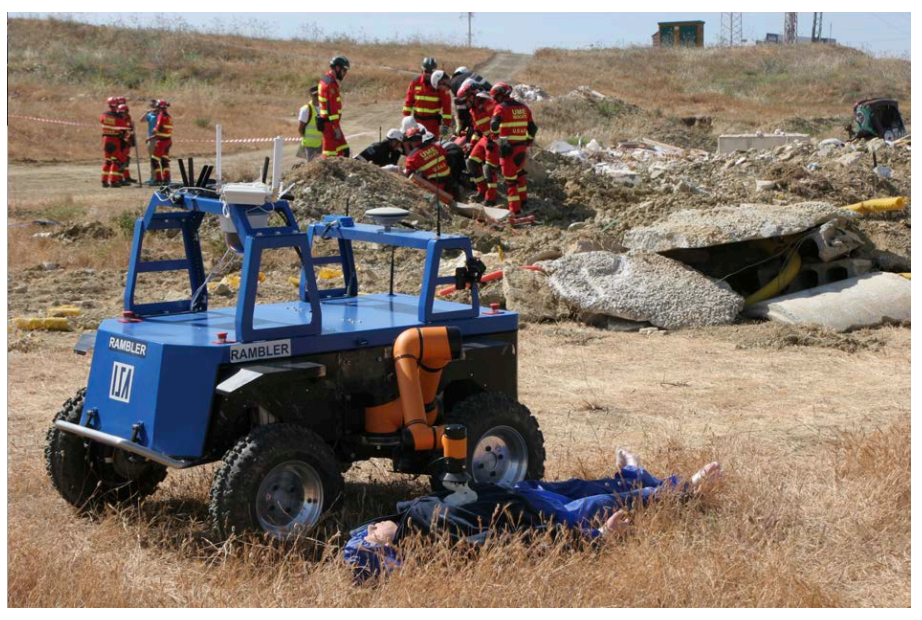

Figura 1: Ejemplo de atención a una víctima con el UGV Rambler durante unas maniobras de respuesta a emergencias. 
ilustra el método mediante un experimento. Finalmente, la sección 5 se dedica a las conclusiones.

\section{TRABAJOS RELACIONADOS}

Los trabajos que han propuesto estrategias de planificación para tareas robóticas relacionadas con misiones de rescate consideran vehículos aéreos no tripulados (UAV, unmanned aerial vehicles), UGV, o combinaciones de ambos.

En [13] se presentó un planificador de vuelo para un UAV mediante puntos de paso para auxiliar a un nadador que emite una señal geolocalizada. Por otra parte, en [8] se propuso la planificación de vuelo con múltiples UAVs para suministro de bienes (medicamentos, comida o agua) a puntos de entrega preestablecidos. En este sentido, en [1] se abordó el problema de la partición de las áreas de trabajo de múltiples UAV en un entorno modelado mediante rejilla.

En cuanto al uso de UGV, en [15] se presentó un control descentralizado que permite guiar un conjunto de vehículos a partir de mapas 3D proporcionados por una formación de UAV. Por otra parte, en [12] se adoptó un enfoque multi-objetivo para resolver el problema del viajante, de modo que un único UGV visite todos los puntos objetivos una sola vez, regresando al punto inicial y con la menor distancia total recorrida. Asimismo, en [4] se aborda la distribución de distintas tareas de respuesta a emergencias para un sistema $\mathrm{UAV} / \mathrm{UGV}$.

La necesidad de atención médica a múltiples víctimas durante una misión SAR, hace necesario adoptar estrategias de planificación multi-objetivo que atiendan a la prioridad de asistencia médica asignada a cada víctima y minimicen el tiempo medio de espera de atención médica. Este tipo de estrategias no se han utilizado en trabajos previos analizados.

\section{PLANIFICADOR ESTRATÉGICO}

El esquema del planificador estratégico para un equipo de múltiples UGV en operaciones de rescate se ilustra en la figura 2. A partir de las características del entorno, los UGV disponibles y las víctimas localizadas, este esquema representa el algoritmo que calcula las trayectorias para el acceso y asistencia a las víctimas, para lo que se considera una representación del entorno en forma de rejilla bidimensional con valores de elevación. Este planificador estratégico se divide en cuatro etapas, que se describen a continuación.
Entradas: entorno, UGVs, víctimas

Calcular mapas de velocidad potencial

Estimar tiempos de desplazamiento

Optimar secuencia para asistencia a víctimas

Determinar trayectorias

Salida: trayectorias

Figura 2: Etapas del planificador estratégico.

\subsection{Etapa 1: Cálculo de mapas de velocidad potencial}

El mapa de velocidad potencial de cada tipo de UGV se construye a partir de las inclinaciones de cada celda del entorno en función de la información de elevación del entorno. Este mapa establece la velocidad proyectada en el plano XY en cada celda para las posibles orientaciones que el UGV puede adoptar en sus desplazamientos. De este modo, para cada celda del entorno, se calcula un vector de ocho elementos correspondientes a cada una de las orientaciones posibles, atendiendo al criterio de 8-conectividad (ángulos múltiplos de $\pi / 4$ radianes $)$.

\subsection{Etapa 2: Estimación de tiempos de desplazamiento}

La estimación de tiempos de desplazamiento hacia y entre víctimas se calcula en función de los mapas de velocidad potencial. Para ello, se propone un nuevo método para entornos anisotrópicos basado en el algoritmo FMM, por el que se propaga un frente de onda desde la posición de cada víctima atendiendo a las velocidades potenciales y orientaciones posibles en los desplazamientos.

El algoritmo original FMM [20] considera un entorno isotrópico, en el que la velocidad de propagación del frente de onda no depende de la dirección adoptada. Sin embargo, esta suposición no es aplicable en espacios anisotrópicos, como por ejemplo aquellos en los que el terreno presenta irregularidades (diferentes inclinaciones atendiendo a las posibles orientaciones del movimiento).

Con el método propuesto se obtiene un mapa de tiempos para cada víctima y tipo de UGV, que 
representa los tiempos de llegada de este tipo de UGV (considerando velocidad de referencia unitaria, $1 \mathrm{~m} / \mathrm{s}$ ) a la víctima desde cada celda del entorno.

A partir de los mapas de tiempo obtenidos se construye una matriz para cada UGV que contiene los tiempos que cada uno invertiría en llegar a cada víctima y entre ellas.

\subsection{Etapa 3: Optimización de secuencias para asistencia a víctimas}

A partir de las matrices de tiempo estimados en la etapa anterior, se establece la secuencia de víctimas que cada UGV deberá asistir. Dicha secuencia de víctimas minimiza el tiempo medio de espera por víctima y atiende a las prioridades de asistencia médica asignadas. De este modo, ninguna asistencia a víctimas incrementará el tiempo medio de espera de víctimas de prioridad superior.

\subsection{Etapa 4: Determinación de trayectorias}

La secuencia de víctimas establecida anteriormente determina una ruta de puntos de paso para cada UGV. La trayectoria del UGV entre dos puntos de paso consecutivos se calcula mediante un descenso de gradiente discreto utilizando el mapa de tiempos previamente obtenido (sección 3.2) así como el mapa de velocidad potencial asociado a cada tipo de UGV.

\section{EJEMPLO DE APLICACIÓN}

Esta sección ilustra la aplicación del planificador estratégico. En particular, se ofrecen resultados para un entorno simulado con tres UGV y siete víctimas.

La figura 3 muestra la representación gráfica del entorno mediante su mapa de elevación. Las dimensiones del mapa son $17 \times 17 \mathrm{~m}$, con una resolución de rejilla de $1 \mathrm{~m}^{2}$. La elevación de cada celda se representa mediante una escala de color (marrón) donde el blanco corresponde a la cota más baja y el negro a valores superiores a $1 \mathrm{~m}$. Los UGV se muestran mediante octógonos de color cian numerados del uno al tres. Las características de los UGV que resultan de interés para el planificador se resumen en la tabla 1. Asimismo, la figura incluye la localización conocida de siete víctimas, indicadas por cuadrados cuyo color corresponde a la prioridad de asistencia médica asignada a cada víctima (triaje). Las distintas prioridades y su correspondiente código de color se definen en la tabla 2 .

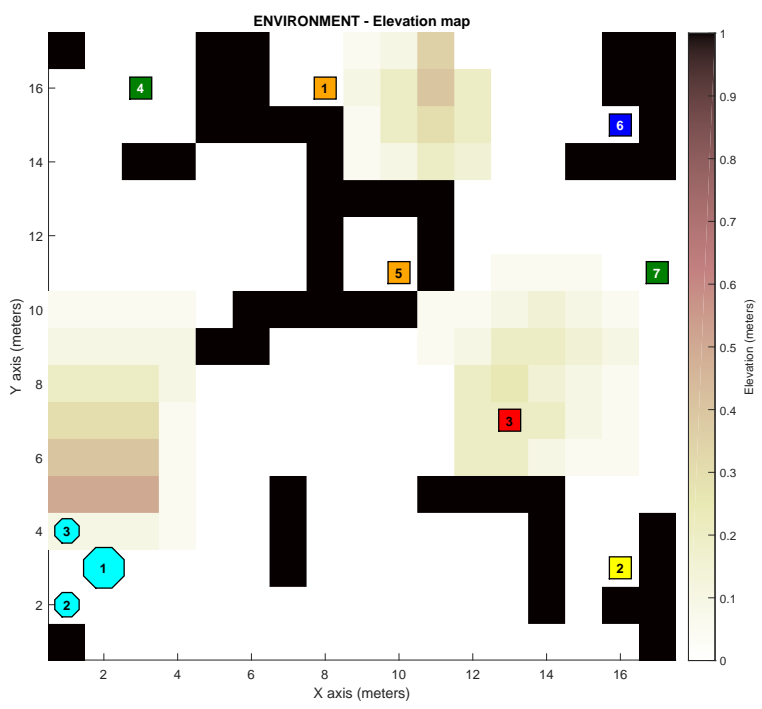

Figura 3: Entorno simulado con tres UGV y siete víctimas.

Tabla 1: Características de los UGV.

\begin{tabular}{lrrr}
\hline & \multicolumn{3}{c}{ ID UGV } \\
\cline { 2 - 4 } & \multicolumn{1}{c}{1} & \multicolumn{1}{c}{2} & \multicolumn{1}{c}{3} \\
\hline Posición inicial $(x, y)(\mathrm{m})$ & $(2,3)$ & $(1,2)$ & $(1,4)$ \\
Dimensiones $(\mathrm{m})$ & $0.4 \times 0.5$ & $0.4 \times 0.5$ & $0.4 \times 0.5$ \\
Radio de seguridad & $0.55 \mathrm{~m}$ & $0.33 \mathrm{~m}$ & $0.33 \mathrm{~m}$ \\
Velocidad desplazamiento & $0.5 \mathrm{~m} / \mathrm{s}$ & $0.1 \mathrm{~m} / \mathrm{s}$ & $0.1 \mathrm{~m} / \mathrm{s}$ \\
Inclinación navegable máxima & $20^{\circ}$ & $10^{\circ}$ & $10^{\circ}$ \\
Inclinación de vuelco lateral & $20^{\circ}$ & $10^{\circ}$ & $10^{\circ}$ \\
\hline
\end{tabular}

Tabla 2: Prioridades de asistencia médica.

\begin{tabular}{ccc}
\hline Nivel & Descripción & Color \\
\hline I & Resucitación & Rojo \\
II & Emergencia & Naranja \\
III & Urgencia & Amarillo \\
IV & Urgencia menor & Verde \\
V & Sin urgencia & Azul \\
O & Fallecido & Negro \\
\hline
\end{tabular}

\subsection{Etapa 1: Cálculo de mapas de velocidad}

En esta etapa se agrupan los UGV que comparten características en relación al radio de seguridad e inclinaciones navegable y antivuelco lateral máxima, constituyendo tipos de UGV a los que se asocia, como velocidad de desplazamiento, una velocidad de referencia unitaria $(1 \mathrm{~m} / \mathrm{s})$. En este entorno simulado, los UGV 2 y 3 comparten dichas características y, por tanto, constituyen un mismo tipo de UGV. Así, para dicho entorno, se definen dos tipos de UGV diferentes.

En las figuras 4 y 5 se muestran los mapas de velocidad potencial correspondientes a cada uno de los tipos de UGV. Las velocidades potenciales determinan la velocidad proyectada en el plano $\mathrm{XY}$ en cada celda del entorno para las posibles 


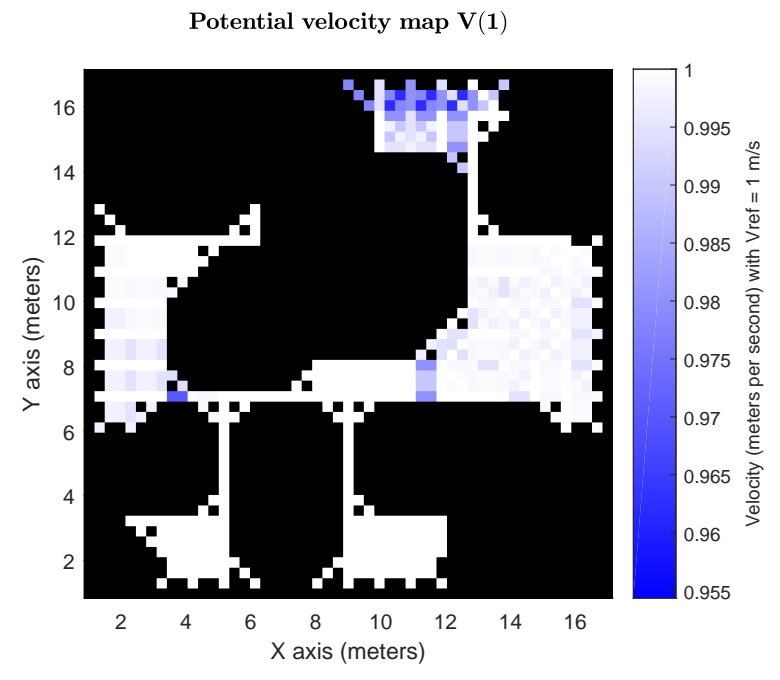

Figura 4: Mapa de velocidad potencial del tipo de $\mathrm{UGV}$ asociado a $\mathrm{UGV}_{1}$.

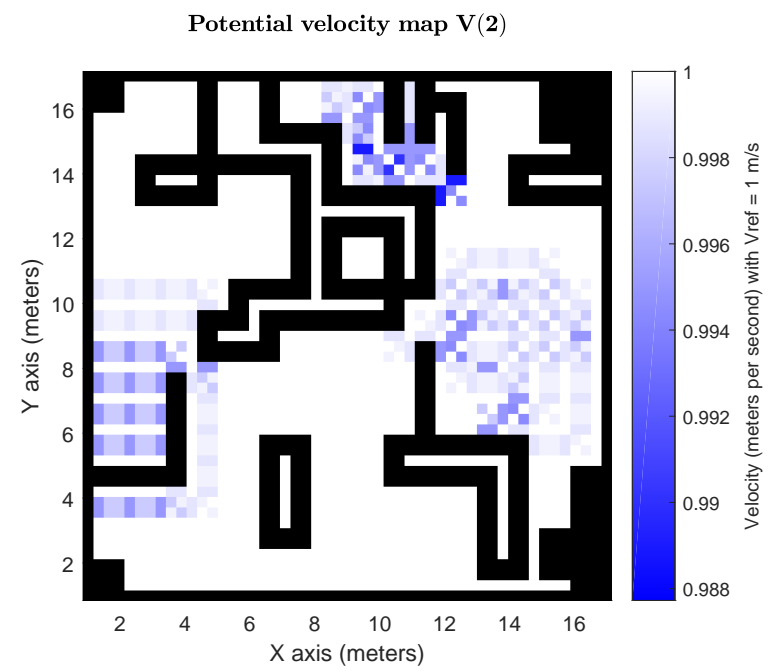

Figura 5: Mapa de velocidad potencial del tipo de $\mathrm{UGV}$ asociado a $\mathrm{UGV}_{2}$ y $\mathrm{UGV}_{3}$.

orientaciones que el UGV puede adoptar en sus desplazamientos. Para ello, se han identificado las orientaciones de desplazamiento no accesibles como aquellas que superan las inclinaciones navegable y antivuelco lateral máximas, asignándoles una velocidad potencial nula. Las velocidades potenciales calculadas se corresponden con una escala de color donde el blanco representa la velocidad máxima (velocidad de referencia unitaria, $1 \mathrm{~m} / \mathrm{s}$ ) y el negro velocidad nula. Mediante tonalidades de azules se representan velocidades, no nulas, inferiores a dicha velocidad de referencia unitaria.

Como se puede observar en las figuras 4 y 5, las áreas del entorno que presentan pendientes tienen asociadas velocidades potenciales inferiores a la velocidad máxima (con colores azules de diferentes intensidades). Los colores negros representan aquellas orientaciones de desplazamientos cuya velocidad potencial es nula y que, por tanto, no están permitidos, evitando así exceder los límites de inclinaciones establecidos como máximos navegable $\mathrm{y}$ antivuelco lateral.

$\mathrm{Al}$ tener el $\mathrm{UGV}_{1}$ un radio de seguridad superior al doble de la resolución de la rejilla, en la figura 4 se ha considerado este radio para dilatar las velocidades potenciales nulas a celdas contiguas, resultando en una mayor área no accesible del entorno.

\subsection{Etapa 2: Estimación de tiempos de desplazamiento}

En esta etapa se estiman los tiempos de desplazamiento de cada tipo de UGV, hacia y entre víctimas. En la figura 6 se representan los mapas de tiempos de llegada para ambos tipos de UGV, asociados a la víctima número 3 . Dichas gráficas representan el tiempo de llegada del UGV a la víctima (considerando velocidad de referencia unitaria, $1 \mathrm{~m} / \mathrm{s}$ ) desde cada celda del entorno. Los tiempos de llegada estimados se corresponden con una escala de color donde el negro representa tiempo infinito y el blanco tiempo nulo. Mediante tonalidades entre amarillos y rojos se representan valores de tiempo, no nulos, inferiores al tiempo máximo de llegada. La posición de la víctima se ha representado como en la figura 3. A través de estos mapas, se puede estimar el tiempo de llegada a la victima desde cualquier posición del entorno, donde áreas de color negro representan posiciones desde donde la víctima no puede ser atendida con dicho tipo de UGV.

A partir de los mapas de tiempos de llegada, como se indicó en la sección (sección 3.2), se construye una matriz para cada UGV que contiene los tiempos de desplazamientos que invertiría el UGV para acceder a cada víctima desde la celda asociada a su posición inicial y desde las celdas correspondientes a las posiciones de cada víctima.

\subsection{Etapa 3: Optimización de secuencias para asistencia a víctimas}

En esta etapa se establece la secuencia de víctimas que cada UGV deberá asistir. Se selecciona, de entre todas las posibles combinaciones de secuencias, aquella que presenta un menor tiempo medio de espera por víctima, atendiendo a las prioridades de asistencia médica asignadas. Para el escenario mostrado en la figura 3 se han evaluado un total de 524.880 secuencias diferentes. 


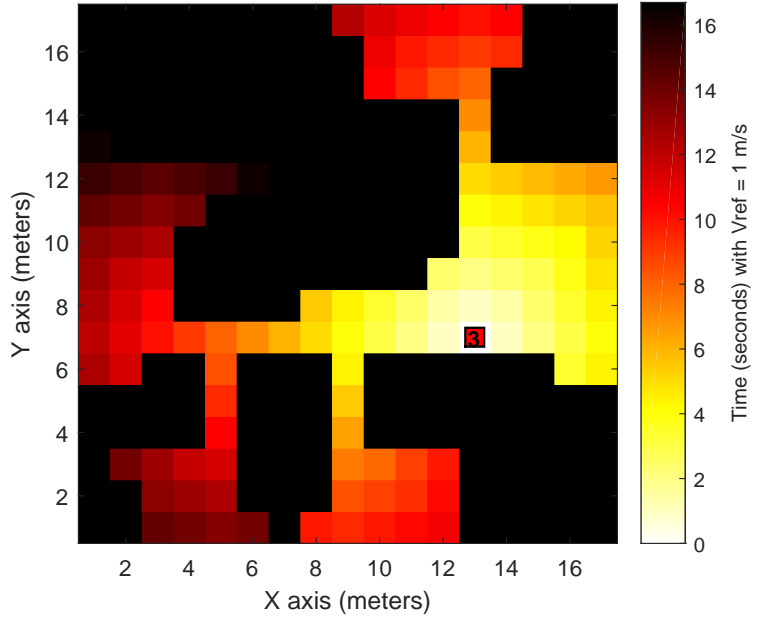

(a) Tipo de agente asociado a $\mathrm{UGV}_{1}$

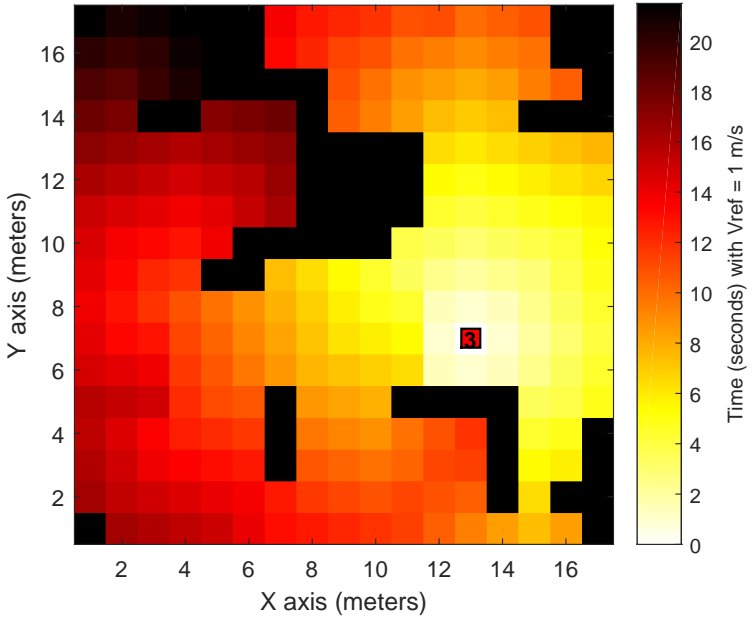

(b) Tipo de agente asociado a $\mathrm{UGV}_{2}$ y $\mathrm{UGV}_{3}$

Figura 6: Mapas de tiempos de llegada a la víctima 3 en el entorno simulado.

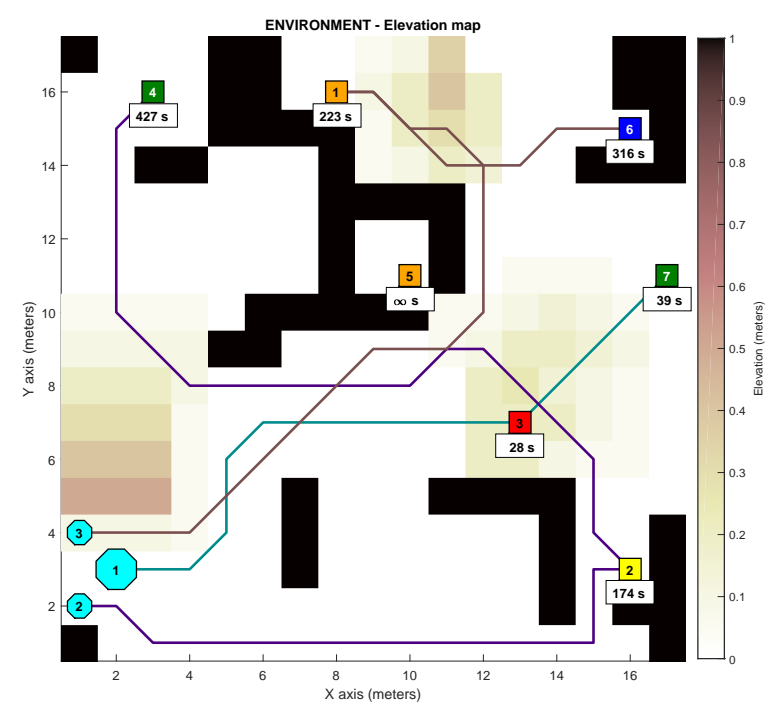

Figura 7: Trayectorias planificadas para el entorno simulado.

\subsection{Etapa 4: Determinación de trayectorias}

Finalmente, en esta etapa se determina la secuencia de celdas que conforman las trayectorias para llevar a cabo la operación de rescate por parte del equipo de UGV. Se realiza un descenso de gradiente discreto sobre los mapas de tiempos de llegada atendiendo a la secuencia de víctimas previamente establecida. En la figura 7 se muestran las trayectorias planificadas para el escenario de la figura 3 , donde bajo la representación de cada víctima se muestra el correspondiente tiempo de espera de asistencia médica.

\section{CONCLUSIONES}

En el presente trabajo se ha presentado un planificador estratégico, que a partir de información aérea previamente obtenida in situ, supone una mayor rapidez en la asistencia a las víctimas, al poder calcular trayectorias de acceso sobre un mapa actualizado del entorno. Dichas trayectorias atienden a las inclinaciones presentes en el terreno, a las características de los UGV disponibles y a la prioridad de asistencia médica asignada a cada víctima. De este modo, se minimiza el tiempo medio de espera de atención médica por víctima, priorizando a aquellas víctimas que así lo requieran. En este sentido se pretende favorecer el incremento de la eficiencia en las operaciones de rescate, ayudando a aumentar el índice de supervivencia.

Como trabajo de desarrollo futuro se proponen realizar mejoras encaminadas a aumentar la seguridad de las trayectorias obtenidas, de modo que éstas se alejen convenientemente de aquellas áreas que supongan un mayor riesgo de vuelco o colisión para los UGV. Asimismo, debido al elevado coste computacional, se propone utilizar métodos de optimización basados en lógica temporal lineal (LTL, linear temporal logic). Estos métodos serán utilizados para establecer la secuencia para asistencia médica que minimice el tiempo medio de espera por víctima, atendiendo a la clasificación de prioridad de asistencia médica asignada.

\section{Agradecimientos}

Este trabajo ha sido financiado por el proyecto nacional DPI2015-65186-R, la Universidad de Málaga y por la ayuda BES-2016-077022 del Fondo Social Europeo FSE. 


\section{English summary}

\section{STRATEGIC PLANNER FOR RES- CUE OPERATIONS WITH UN- MANNED GROUND VEHICLES}

\begin{abstract}
The deployment of robotic platforms in support of emergency management is a challange that seeks to improve efficiency in search and rescue missions. This paper proposes a strategic planner for the rescue of victims with a team of ground robots. The purpose of this intelligent system is planning the routes to access a number of victims according to their location and priority of medical assistance (i.e., triage). In particular, this paper offers work in progress regarding a new anisotropic variant of the fast marching method (FMM). Furthermore, preliminary results illustrate the application under simulated conditions.
\end{abstract}

Keywords: Strategic planner, rescue operations, fast marching method, unmanned ground vehicles, triage

\section{Referencias}

[1] Acevedo, J. J., Arrue, B. C., Maza, I. and Ollero, A.: 2016, A distributed algorithm for area partitioning in grid-shape and vectorshape configurations with multiple aerial robots, Journal of Intelligent and Robotic Systems: Theory and Applications 84(1-4), 543557.

[2] Baca, J., Ferre, M., Campos, A., Fernandez, J. and Aracil, R.: 2010, On the analysis of a multi-task modular robot system for field robotics, pp. 475-480.

[3] Baizid, K., Giglio, G., Pierri, F., Trujillo, M., Antonelli, G., Caccavale, F., Viguria, A., Chiaverini, S. and Ollero, A.: 2017, Behavioral control of unmanned aerial vehicle manipulator systems, Autonomous Robots 41(5), 1203-1220.

[4] Bermejo-Alonso, J., Salvador, J. and Sanz, R.: 2018, Towards an ontology for task and planning in autonomous systems: An emergency scenario, Advances in Intelligent Systems and Computing 693, 429-440.
[5] Bernard, M., Kondak, K., Maza, I. and Ollero, A.: 2011, Autonomous transportation and deployment with aerial robots for search and rescue missions, Journal of Field Robotics 28(6), 914-931.

[6] Breñosa, J., Cerrada, P., Ferre, M. and Aracil, R.: 2011, Design of an ergonomic threefinger haptic device for advanced robotic hands control, pp. 257-262.

[7] De León, J., Garzón, M., Garzón-Ramos, D. and Barrientos, A.: 2018, Study of gait patterns for an hexapod robot in search and rescue tasks, Advances in Intelligent Systems and Computing 694, 731-742.

[8] Doherty, P. and Rudol, P.: 2017, A UAV search and rescue scenario with human body detection and geolocalization, Advanced in Artificial Intelligence, AI. Lecture Notes in Computer Science 4830, 1-13.

[9] Escribano, J. G., García, A. and de la Fuente, M.: 2011, Monitorización de la condición física de personas en espacios confinados mediante etiquetas RFID con sensores y redes inalámbricas eficientes, Revista Iberoamericana de Automática e Informática Industrial 8(4), $371-384$.

[10] Guzman, R., Navarro, R., Ferre, J. and Moreno, M.: 2016, Rescuer: Development of a modular chemical, biological, radiological, and nuclear robot for intervention, sampling, and situation awareness*, Journal of Field Robotics 33(7), 931-945.

[11] Kostabelils, I. and Gasteratos, A.: 2017, Robots in crisis management: A survey, International Conference on Information Systems for Crisis Response and Management in Mediterranean Countries pp. 43-56.

[12] Luo, C., Shen, F., Yang, S. X. and Mo, H.: 2015, Annealing-based guidance of a rescue robot for rescue mission with multi-goal navigation, IEEE International Conference on Information and Automation pp. 861-866.

[13] Lygouras, E., Gasteratos, A. and Tarchanidis, K.: 2017, ROLFER: An innovative proactive platform to reserve swimmer's safety, International Conference on Information Systems for Crisis Response and Management in Mediterranean Countries pp. 57-69.

[14] Murphy, R. R., Tadokoro, S. and Kleiner, A.: 2016, Springer Handbook of Robotics, 2 edn, Springer, chapter 60, Disaster Robotics, pp. 1577-1604.

[15] Neto, A. A., Macharet, D. G. and Campos, M. F. M.: 2018, Multi-agent rapidly- 
exploring pseudo-random tree, Journal of Intelligent \& Robotic Systems 89(1-2), 69-85.

[16] Nuño, E., Valle, D., Sarras, I. and Basanez, L.: 2013, Bilateral teleoperation of flexible-joint manipulators with dynamic gravity compensation and variable time-delays, pp. 5439-5444.

[17] Papadakis, P.: 2013, Terrain traversability analysis methods for unmanned ground vehicles: A survey, Engineering Applications of Artificial Intelligence 26(4), 1373-1385.

[18] Pomares, J., Perea, I. and Torres, F.: 2014, Dynamic visual servoing with chaos control for redundant robots, IEEE/ASME Transactions on Mechatronics 19(2), 423-431.

[19] Portilla, H. and Basañez, L.: 2007, Augmented reality tools for enhanced robotics teleoperation systems.

[20] Sethian, J.: 1996, A fast marching level set method for monotonically advancing fronts,
Proceedings of the National Academy of Sciences of the United States of America 93(4), 1591-1595.

[21] Tardioli, D., Sicignano, D., Riazuelo, L., Romeo, A., Villarroel, J. L. and Montano, L.: 2016, Robot teams for intervention in confined and structured environments, Journal of Field Robotics 33(6), 765-801.

[22] Youakim, D. and Ridao, P.: 2018, Motion planning survey for autonomous mobile manipulators underwater manipulator case study, Robotics and Autonomous Systems 107, 20-44.

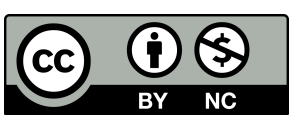

(C) 2018 by the authors. Submitted for possible open access publication under the terms and conditions of the Creative Commons Attribution CC-BY-NC 3.0 license (http://creativecommons.org/licenses/by-nc/3.0/). 